\title{
BOSONS IN A TRAP: ASYMPTOTIC EXACTNESS OF THE GROSS-PITAEVSKII GROUND STATE ENERGY FORMULA
}

\author{
ROBERT SEIRINGER
}

\begin{abstract}
Recent experimental breakthroughs in the treatment of dilute Bose gases have renewed interest in their quantum mechanical description, respectively in approximations to it. The ground state properties of dilute Bose gases confined in external potentials and interacting via repulsive short range forces are usually described by means of the Gross-Pitaevskii energy functional. In joint work with Elliott $\mathrm{H}$. Lieb and Jakob Yngvason its status as an approximation for the quantum mechanical many-body ground state problem has recently been rigorously clarified. We present a summary of this work, for both the two- and three-dimensional case.
\end{abstract}

\section{INTRODUCTION}

The Gross-Pitaevskii (GP) functional was introduced in the early sixties as a phenomenological description of the order parameter in superfluid $\mathrm{He}_{4}$ G61, P61, G63. It has come into prominence again because of recent experiments on BoseEinstein condensation of dilute gases in magnetic traps. The paper [DGPS99] brings an up to date review of these developments.

The present contribution is based on the joint work [LSY00a, LSY00b with Elliott H. Lieb and Jakob Yngvason (see also [LSY00d). The starting point of our investigation is the Hamiltonian for $N$ identical bosons moving in $\mathbb{R}^{D}, D=2$ or 3 , that interact with each other via a radially symmetric pair-potential $v\left(\left|\vec{x}_{i}-\vec{x}_{j}\right|\right)$ and are confined by an external potential $V(\vec{x})$ :

$$
H=\sum_{i=1}^{N}\left\{-\Delta_{i}+V\left(\vec{x}_{i}\right)\right\}+\sum_{1 \leq i<j \leq N} v\left(\left|\vec{x}_{i}-\vec{x}_{j}\right|\right) .
$$

The Hamiltonian acts on symmetric wave functions in $\otimes^{N} L^{2}\left(\mathbb{R}^{D}, d \vec{x}\right)$. The pair interaction $v$ is assumed to be nonnegative and of short range, more precisely, we demand it to have a finite scattering length. (For a definition of the scattering length in arbitrary dimension see [LY00.) The potential $V$ that represents the trap is locally bounded and $V(\vec{x}) \rightarrow \infty$ as $|\vec{x}| \rightarrow \infty$. By shifting the energy scale we can assume that $\min _{\vec{x}} V(\vec{x})=0$.

Units are chosen so that $\hbar=2 m=1$, where $m$ is the particle mass. A natural energy unit is given by the ground state energy $\hbar \omega$ of the one particle Hamiltonian $-\left(\hbar^{2} / 2 m\right) \Delta+V$. The corresponding length unit, $\sqrt{\hbar /(m \omega)}$, measures the effective extension of the trap.

1991 Mathematics Subject Classification. Primary 81V70; Secondary 35Q55, 46N50. 
We are interested in the ground state energy $E^{\mathrm{QM}}=\inf \operatorname{spec} H$. Besides $N$ it depends on the potentials $V$ and $v$, but with $V$ fixed and

$$
v(r)=\left(a_{1} / a\right)^{2} v_{1}\left(a_{1} r / a\right),
$$

where $v_{1}$ has scattering length $a_{1}$ and is regarded as fixed, $E^{\mathrm{QM}}$ is a function of $N$ and $a$ only. The corresponding ground state density is given by

$$
\rho^{\mathrm{QM}}(\vec{x})=N \int\left|\Psi_{0}\left(\vec{x}, \vec{x}_{2}, \ldots, \vec{x}_{N}\right)\right|^{2} d \vec{x}_{2} \ldots d \vec{x}_{N},
$$

where $\Psi_{0}$ is a ground state wave function of $H$.

Note that $v$ given in (2) has scattering length $a$. Here $a$ is dimensionless and really stands for $a \sqrt{m \omega / \hbar}$. Hence a scaling of $v$ like (2) is equivalent to scaling the external potential $V$ at fixed $v$. In particlar the limit $a \rightarrow 0$ with fixed $V$ is equivalent to the limit $\omega \rightarrow 0$ with fixed $v$, if one introduces the scaling $V(\vec{x})=\omega V_{1}\left(\omega^{1 / 2} \vec{x}\right)$ for some fixed $V_{1}$.

Recent experiments on Bose-Einstein condensation are usually interpreted in terms of a function $\Phi^{\mathrm{GP}}(\vec{x})$ of $\vec{x} \in \mathbb{R}^{D}$, which minimizes the Gross-Pitaevskii energy functional

$$
\mathcal{E}^{\mathrm{GP}}[\Phi]=\int_{\mathbb{R}^{D}}\left(|\nabla \Phi|^{2}+V|\Phi|^{2}+4 \pi g|\Phi|^{4}\right) d \vec{x}
$$

under the subsidiary condition $\int|\Phi|^{2}=N$. The corresponding energy is

$$
E^{\mathrm{GP}}(N, g)=\inf _{\int|\Phi|^{2}=N} \mathcal{E}^{\mathrm{GP}}[\Phi]=\mathcal{E}^{\mathrm{GP}}\left[\Phi^{\mathrm{GP}}\right] .
$$

The parameter $g$ is different in dimensions 2 and 3. However, for any value of $g>0$ and $N>0$ it can be shown that a unique, strictly positive $\Phi^{\mathrm{GP}}$ exists LSY00a. It depends on these parameters, of course, and when this is important we denote it by $\Phi_{N, g}^{\mathrm{GP}}$.

The motivation of the term $4 \pi g|\Phi|^{4}$ in the GP functional comes from the ground state energy density, $\varepsilon_{0}(\rho)$, of a dilute, thermodynamically infinite, homogeneous Bose gas of density $\rho$, interacting via a repulsive potential with scattering length $a$. The formulas for this quantity are older than the GP functional B47, HY57, S71, at least for $D=3$, but they have only very recently been derived rigorously for suitable interparticle potentials. See [LY8] and LY00]. They are given by

$$
\begin{aligned}
& \varepsilon_{0}(\rho) \approx 4 \pi a \rho^{2} \quad \text { for } D=3, \\
& \varepsilon_{0}(\rho) \approx 4 \pi \rho^{2}\left|\ln \left(a^{2} \rho\right)\right|^{-1} \quad \text { for } D=2,
\end{aligned}
$$

where $\approx$ means that the formulas are valid for dilute gases, where $a^{D} \rho \ll 1$. Hence the natural choice of the parameter $g$ is

$$
\begin{aligned}
& g=a \quad \text { for } D=3, \\
& g=\left|\ln \left(a^{2} \bar{\rho}\right)\right|^{-1} \quad \text { for } D=2,
\end{aligned}
$$

where $\bar{\rho}$ is the mean GP density

$$
\bar{\rho}=\frac{1}{N} \int\left|\Phi^{\mathrm{GP}}(\vec{x})\right|^{4} d \vec{x} .
$$

Note that $\Phi^{\mathrm{GP}}$ depends on $g$, so (8) together with (9) are non-linear equations for $g$. Alternatively, one could define $g$ using the minimizer for $g=1$ in the definition of $\bar{\rho}$. Since $\bar{\rho}$ appears only under a logarithm, this would not effect our leading order calculations. For the same reason one could use the TF minimizer 
(see below) instead of the GP minimizer to define $g$. Note also that unlike in the three-dimensional case $g$ depends on $N$ in the two-dimensional case.

The idea is now that with this choice of $g$ one should, for dilute gases, have that

$$
E^{\mathrm{GP}} \approx E^{\mathrm{QM}} \quad \text { and } \quad \rho^{\mathrm{QM}}(\vec{x}) \approx\left|\Phi^{\mathrm{GP}}(\vec{x})\right|^{2} \equiv \rho^{\mathrm{GP}}(\vec{x}) .
$$

This is made precise in the following theorems. Note that by scaling

$$
E^{\mathrm{GP}}(N, g)=N E^{\mathrm{GP}}(1, N g) \quad \text { and } \quad \Phi_{N, g}^{\mathrm{GP}}(\vec{x})=N^{1 / 2} \Phi_{1, N g}^{\mathrm{GP}}(\vec{x}) .
$$

Hence $N g$ is the natural parameter in GP theory. With this in mind we can state our first main result.

Theorem 1.1 (The GP limit of the QM ground state energy and density). If $N \rightarrow \infty$ with $\mathrm{Ng}$ fixed, then

$$
\lim _{N \rightarrow \infty} \frac{E^{\mathrm{QM}}(N, a)}{E^{\mathrm{GP}}(N, g)}=1,
$$

and

$$
\lim _{N \rightarrow \infty} \frac{1}{N} \rho^{\mathrm{QM}}(\vec{x})=\left|\Phi_{1, N g}^{\mathrm{GP}}(\vec{x})\right|^{2}
$$

in the weak $L_{1}$-sense.

Note that by hypothesis of the theorem above it really applies to dilute gases, since for fixed $N g$ (which we refer to as the GP case) the mean density $\bar{\rho}$ is of order $N$ and

$$
a^{3} \bar{\rho} \sim N^{-2} \quad \text { for } \quad D=3, \quad a^{2} \bar{\rho} \sim \exp (-N) \quad \text { for } \quad D=2 .
$$

Especially for $D=2$ this is an unsatisfactory restriction, since $a$ has to decrease exponentially with $N$. For a slower decrease $N g$ tends to infinity with $N$, and the same holds for $D=3$ if $a$ does not decrease at least as $N^{-1}$. In this case, the gradient term in the GP functional becomes negligible compared to the other terms and the so-called Thomas-Fermi (TF) functional

$$
\mathcal{E}^{\mathrm{TF}}[\rho]=\int_{\mathbb{R}^{D}}\left(V \rho+4 \pi g \rho^{2}\right) d \vec{x}
$$

arises. It is defined for nonnegative functions $\rho$ on $\mathbb{R}^{D}$. Its ground state energy $E^{\mathrm{TF}}$ and density $\rho^{\mathrm{TF}}$ are defined analogously to the GP case. Our second main result is that minimization of (15) reproduces correctly the ground state energy and density of the many-body Hamiltonian in the limit when $N \rightarrow \infty, a^{D} \bar{\rho} \rightarrow 0$, but $N g \rightarrow \infty$ (which we refer to as the TF case), provided the external potential is reasonably well behaved. We will assume that $V$ is asymptotically equal to some function $W$ that is homogeneous of some order $s>0$ and locally Hölder continuous (see LSY00b for a precise definition). This condition can be relaxed, but it seems adequate for most practical applications and simplifies things considerably.

Theorem 1.2 (The TF limit of the QM ground state energy and density). Assume that $V$ satisfies the conditions stated above. If $\gamma \equiv N g \rightarrow \infty$ as $N \rightarrow \infty$, but still $a^{D} \bar{\rho} \rightarrow 0$, then

$$
\lim _{N \rightarrow \infty} \frac{E^{\mathrm{QM}}(N, a)}{E^{\mathrm{TF}}(N, g)}=1
$$


and

$$
\lim _{N \rightarrow \infty} \frac{\gamma^{D /(s+D)}}{N} \rho^{\mathrm{QM}}\left(\gamma^{1 /(s+D)} \vec{x}\right)=\tilde{\rho}^{\mathrm{TF}}(\vec{x})
$$

in the weak $L_{1}$-sense, where $\tilde{\rho}^{\mathrm{TF}}$ is the minimizer of the TF functional under the condition $\int \rho=1, g=1$, and with $V$ replaced by $W$.

Remark. The theorems are independent of the interaction potential $v_{1}$ in (2). This means that in the limit we consider only the scattering length effects the ground state properties, and not the details of the potential. Note also that the particular limit we consider is not a mean field limit, since the interaction potential is very hard in this limit; in fact the term $4 \pi g|\Phi|^{4}$ is mostly kinetic energy.

In the following, we will present a brief sketch of the proof of Theorems 1.1 and 1.2. We will derive appropriate upper and lower bounds on the ground state energy $E^{\mathrm{QM}}$. The convergence of the densities follows from the convergence of the energies in the usual way by variation with respect to the external potential. We refer to LSY00a and LSY00b for details.

\section{UPPER BOUND TO THE QM ENERGY}

To derive an upper bound on $E^{\mathrm{QM}}$ we use a generalization of a trial wave function of Dyson [D57], who used this function to give an upper bound on the ground state energy of the homogeneous hard core Bose gas. It is of the form

$$
\Psi\left(\vec{x}_{1}, \ldots, \vec{x}_{N}\right)=\prod_{i=1}^{N} \Phi^{\mathrm{GP}}\left(\vec{x}_{i}\right) F\left(\vec{x}_{1}, \ldots, \vec{x}_{N}\right),
$$

where $F$ is constructed in the following way:

$$
F\left(\vec{x}_{1}, \ldots, \vec{x}_{N}\right)=\prod_{i=1}^{N} f\left(t_{i}\left(\vec{x}_{1}, \ldots, \vec{x}_{i}\right)\right),
$$

where $t_{i}=\min \left\{\left|\vec{x}_{i}-\vec{x}_{j}\right|, 1 \leq j \leq i-1\right\}$ is the distance of $\vec{x}_{i}$ to its nearest neighbor among the points $\vec{x}_{1}, \ldots, \vec{x}_{i-1}$, and $f$ is a function of $r \geq 0$. We choose it to be

$$
f(r)=\left\{\begin{array}{cl}
f_{0}(r) / f_{0}(b) & \text { for } \quad r<b \\
1 & \text { for } \quad r \geq b
\end{array}\right.
$$

where $f_{0}$ is the solution of the zero-energy scattering equation (see [LY00) and $b$ is some cut-off parameter of order $b \sim \bar{\rho}^{-1 / D}$. The function (18) is not totally symmetric, but for an upper bound it is nevertheless an acceptable test wave function since the bosonic ground state energy is equal to the absolute ground state energy.

The result of a somewhat lengthy computation is the upper bound

$$
E^{\mathrm{QM}}(N, a) \leq E^{\mathrm{GP}}(N, g) \times \begin{cases}1+O\left(a \bar{\rho}^{1 / 3}\right) & \text { for } \quad D=3 \\ 1+O\left(\left|\ln \left(a^{2} \bar{\rho}\right)\right|^{-p}\right) & \text { for } \quad D=2,\end{cases}
$$

with the power $p$ equal to 1 in the GP case and $s /(s+2)$ in the TF case (where $V$ is asymptotically homogeneous of order $s$ ). 


\section{LOWER BOUND TO THE QM ENERGY}

To obtain a lower bound for the QM energy the strategy is to divide space into boxes and use the estimate on the homogeneous gas, given in [LY98] and [LY00], in each box with Neumann boundary conditions. One then minimizes over all possible divisions of the particles among the different boxes. This gives a lower bound to the energy because discontinuous wave functions for the quadratic form defined by the Hamiltonian are now allowed. We can neglect interactions among particles in different boxes because $v \geq 0$. Finally, one lets the box size tend to zero. However, it is not possible to simply approximate $V$ by a constant potential in each box. To see this consider the case of noninteracting particles, i.e., $v=0$ and hence $a=0$. Here $E^{\mathrm{QM}}=N \hbar \omega$, but a 'naive' box method gives only 0 as lower bound, since it clearly pays to put all the particles with a constant wave function in the box with the lowest value of $V$.

For this reason we start by separating out the GP wave function in each variable and write a general wave function $\Psi$ as

$$
\Psi\left(\vec{x}_{1}, \ldots, \vec{x}_{N}\right)=\prod_{i=1}^{N} \Phi^{\mathrm{GP}}\left(\vec{x}_{i}\right) F\left(\vec{x}_{1}, \ldots, \vec{x}_{N}\right) .
$$

This defines $F$ for a given $\Psi$ because $\Phi^{\mathrm{GP}}$ is everywhere strictly positive, being the ground state of the operator $-\Delta+V+8 \pi g\left|\Phi^{\mathrm{GP}}\right|^{2}$. We now compute the expectation value of $H$ in the state $\Psi$. Using partial integration and the variational equation for $\Phi^{\mathrm{GP}}$, we see that for computing the ground state energy of $H$ we have to minimize the normalized quadratic form

$$
Q(F)=\sum_{i=1}^{N} \frac{\int \prod_{k=1}^{N} \rho^{\mathrm{GP}}\left(\vec{x}_{k}\right)\left(\left|\nabla_{i} F\right|^{2}+\sum_{j=1}^{i-1} v\left(\left|\vec{x}_{i}-\vec{x}_{j}\right|\right)|F|^{2}-8 \pi g \rho^{\mathrm{GP}}\left(\vec{x}_{i}\right)|F|^{2}\right)}{\int \prod_{k=1}^{N} \rho^{\mathrm{GP}}\left(\vec{x}_{k}\right)|F|^{2}} .
$$

Compared to the expression for the energy involving $\Psi$ itself we have thus obtained the replacements

$$
V(\vec{x}) \rightarrow-8 \pi g \rho^{\mathrm{GP}}(\vec{x}) \quad \text { and } \quad \prod_{i=1}^{N} d \vec{x}_{i} \rightarrow \prod_{i=1}^{N} \rho^{\mathrm{GP}}\left(\vec{x}_{i}\right) d \vec{x}_{i}
$$

(recall that $\rho^{\mathrm{GP}}(\vec{x})=\left|\Phi^{\mathrm{GP}}(\vec{x})\right|^{2}$ ). We now use the box method on this problem. More precisely, labeling the boxes by an index $\alpha$, we have

$$
\inf _{F} Q(F) \geq \inf _{\left\{n_{\alpha}\right\}} \sum_{\alpha} \inf _{F_{\alpha}} Q_{\alpha}\left(F_{\alpha}\right),
$$

where $Q_{\alpha}$ is defined by the same formula as $Q$ but with the integrations limited to the box $\alpha, F_{\alpha}$ is a wave function with particle number $n_{\alpha}$, and the infimum is taken over all distributions of the particles with $\sum n_{\alpha}=N$. Approximating $\rho^{\mathrm{GP}}$ by a constant in each box, we can use the bound on the homogeneous case (LY98 and [LY00]) in each box. To control the error terms, we need a lower bound on the ratio $\rho_{\alpha, \min } / \rho_{\alpha, \max }$ in each box, which stems from the measures in (23), where $\rho_{\alpha, \max }$ and $\rho_{\alpha, \min }$, respectively, denote the maximal and minimal values of $\rho^{\mathrm{GP}}$ in box $\alpha$. 
The problem is that for any fixed size of boxes $\rho_{\alpha, \min } / \rho_{\alpha, \max }$ tends rapidly to zero for boxes far from the origin. This problem can be solved by enclosing the whole system in a big box $\Lambda_{R}$ of side length $R$, with Neumann conditions on the boundary. Replacing $\Phi^{\mathrm{GP}}$ by $\Phi_{R}^{\mathrm{GP}}$, which is the minimizer of $\mathcal{E}^{\mathrm{GP}}$ restricted to $\Lambda_{R}$ (and satisfies Neumann conditions), and restricting the integrations to $\Lambda_{R}$ we can let the side lengths of the small boxes tend to zero and be sure that $\rho_{\alpha, \min } / \rho_{\alpha, \max } \rightarrow 1$ uniformly for all the boxes $\alpha$. However, we must control the error made by enclosing the system in the big box. Let $E_{R}^{\mathrm{QM}}(N, a)$ denote the quantum mechanical ground state energy in the box $\Lambda_{R}$. The essential step is

Lemma 3.1. There is an $R_{0}<\infty$, depending only on $N g$, such that

$$
E^{\mathrm{QM}}(N, a) \geq E_{R}^{\mathrm{QM}}(N, a)
$$

for all $R \geq R_{0}$ and all $N$, a with $N g$ fixed.

This lemma follows from $V(\vec{x}) \rightarrow \infty$ for $|\vec{x}| \rightarrow \infty$, together with an estimate for the chemical potential

$$
E_{R}^{\mathrm{QM}}(N+1, a)-E_{R}^{\mathrm{QM}}(N, a) \leq e(N g)(1+o(1))
$$

where $e(N g)$ depends only on $N g$ and is independent of $R$. The proof of (27) is similar to the proof of the upper bound (21).

Now $\rho_{\alpha, \min } / \rho_{\alpha, \max }$ is bounded below uniformly in each small box contained in $\Lambda_{R}$. We first let the size of the boxes tend to zero as $N \rightarrow \infty$, and finally take the limit $R \rightarrow \infty$. This proves the desired lower bound (for fixed $N g$, i.e. the GP case).

If $N g \rightarrow \infty$ as $N \rightarrow \infty$ the method above does not work since the $R_{0}$ of Lemma 3.1 tends to infinity with $N g$. However, using the explicit form of the TF minimizer, namely

$$
\rho_{N, g}^{\mathrm{TF}}(\vec{x})=\frac{1}{8 \pi g}\left[\mu^{\mathrm{TF}}-V(\vec{x})\right]_{+},
$$

where $[t]_{+} \equiv \max \{t, 0\}$ and $\mu^{\mathrm{TF}}$ is chosen so that the normalization condition $\int \rho_{N, g}^{\mathrm{TF}}=N$ holds, we can use $V(\vec{x}) \geq \mu^{\mathrm{TF}}-8 \pi g \rho^{\mathrm{TF}}(\vec{x})$ to get an replacement as in (24) without changing the measure. Moreover, $\rho^{\mathrm{TF}}$ has compact support, so, applying again the box method described above, the boxes far out do not contribute to the energy. However, $\mu^{\mathrm{TF}}$ (which depends only on the combination $N g$ ) tends to infinity as $N g \rightarrow \infty$. Therefore we need to control the asymptotic behaviour of the external potential, and this leads to the restrictions on $V$ described in the paragraph preceding Theorem 1.2 .

After controlling all error terms properly, we arrive at the result

$$
\liminf _{N \rightarrow \infty} \frac{E^{\mathrm{QM}}(N, a)}{E^{\mathrm{TF}}(N, g)} \geq 1
$$

in the limit $N \rightarrow \infty, a^{D} \bar{\rho} \rightarrow 0$ and $N g \rightarrow \infty$. Together with the upper bound (21) and the fact that $E^{\mathrm{GP}}(N, g) / E^{\mathrm{TF}}(N, g)=E^{\mathrm{GP}}(1, N g) / E^{\mathrm{TF}}(1, N g) \rightarrow 1$ as $N g \rightarrow \infty$ this proves Theorem 1.2 .

\section{REFERENCES}

[B47] N.N. Bogoliubov, J. Phys. (U.S.S.R.) 11, 23 (1947); N.N. Bogoliubov and D.N. Zubarev, Sov. Phys.-JETP 1, 83 (1955).

[D57] F.J. Dyson, Ground-State Energy of a Hard-Sphere Gas, Phys. Rev. 106, 20-26 (1957). 
[DGPS99] F. Dalfovo, S. Giorgini, L.P. Pitaevskii, and S. Stringari, Theory of Bose-Einstein condensation in trapped gases, Rev. Mod. Phys. 71, 463-512 (1999).

[G61] E.P. Gross, Structure of a Quantized Vortex in Boson Systems, Nuovo Cimento 20, 454-466 (1961).

[G63] E.P. Gross, Hydrodynamics of a superfluid condensate, J. Math. Phys. 4, 195-207 (1963).

[HY57] K. Huang, C.N. Yang, Phys. Rev. 105, 767-775 (1957); T.D. Lee, K. Huang, and C.N. Yang, Phys. Rev. 106, 1135-1145 (1957); K.A. Brueckner, K. Sawada, Phys. Rev. 106, 1117-1127, 1128-1135 (1957); S.T. Beliaev, Sov. Phys.-JETP 7, 299-307 (1958); T.T. Wu, Phys. Rev. 115, 1390 (1959); N. Hugenholtz, D. Pines, Phys. Rev. 116, 489 (1959); M. Girardeau, R. Arnowitt, Phys. Rev. 113, 755 (1959); T.D. Lee, C.N. Yang, Phys. Rev. 117, 12 (1960); E.H. Lieb, Phys. Rev. 130, 2518-2528 (1963).

[LSY00a] E.H. Lieb, R. Seiringer, and J. Yngvason, Bosons in a trap: a rigorous derivation of the Gross-Pitaevskii energy functional, Phys. Rev. A 61, 043602-1-13 (2000).

[LSY00b] E.H. Lieb, R. Seiringer, and J. Yngvason, A rigorous derivation of the Gross-Pitaevskii energy functional for a two-dimensional Bose gas, arXiv: cond-mat/0005026, to appear in Commun. Math. Phys.

[LSY00c] E.H. Lieb, R. Seiringer, and J. Yngvason, The Ground State Energy and Density of Interacting Bosons in a Trap, in Quantum Theory and Symmetries, Goslar, 1999, H.-D. Doebner, V.K. Dobrev. J.-D. Hennig and W. Luecke, eds., pp. 101-110, World Scientific (2000). arXiv math-ph/9911026, mp_arc 99-439.

[LY98] E.H. Lieb and J. Yngvason, Ground state energy of the low density Bose gas, Phys. Rev. Lett. 80, 2504-2507 (1998).

[LY00] E.H. Lieb and J. Yngvason, Ground State Energy of a Dilute Two-dimensional Bose Gas, arXiv: math-ph/0002014, J. Stat. Phys. (in press)

[P61] L.P. Pitaevskii, Vortex lines in an imperfect Bose gas, Sov. Phys. JETP, 13, 451-454 (1961).

[S71] M. Schick, Two-Dimensional System of Hard Core Bosons, Phys. Rev. A 3, 1067-1073 (1971).

Institut für Theoretische Physik, Universität Wien, Boltzmanngasse 5, A-1090 ViENNA, AUSTRIA

E-mail address: rseiring@ap.univie.ac.at 